\title{
Research on the Evaluation of Teaching Quality Based on CGSAB
}

\author{
Jiatang Cheng* \\ The Engineering College \\ Honghe University \\ Mengzi 661199, China
}

\author{
Yan Xiong \\ The Engineering College \\ Honghe University \\ Mengzi 661199, China
}

\begin{abstract}
Teaching quality evaluation is an important work in teaching management. In order to improve the accuracy of teaching quality evaluation, according to the evaluation data at a certain university, an evaluation model based on BP neural network optimized by gravitational search algorithm (GSABP) is proposed. For the GSA algorithm is easy to fall into the local optimal, the ergodicity of chaotic sequence is used to generate the initial population of GSA, and then the chaotic gravitational search algorithm (CGSA) is presented. The experimental results show that, compared with BP neural network and GSABP algorithm, the model using CGSABP has high credibility and strong generalization ability, which provides a feasible method for the accurate evaluation of teaching quality.
\end{abstract}

Keywords-teaching quality; evaluation; gravitational search algorithm; chaotic series; BP neural network

\section{INTRODUCTION}

Teaching quality is the fundamentality of the existence of colleges and universities, the evaluation of teachers' teaching quality is the normal means adopted by the school in order to guarantee the high quality teaching effect [1]. In recent years, many colleges and universities have established the teaching quality assessment systems, which hope to improve the teaching quality through a series of measures. Teaching quality evaluation refers to the use of scientific and feasible evaluation methods in a specific educational goal, to make judgments on the value of teaching to improve teaching, enhance teaching quality and provide a reliable information and scientific basis [2].

In order to reflect the scientific nature, comprehensiveness, accuracy and maneuverability of classroom teaching quality evaluation, according to the evaluation index system of teaching work of the ordinary colleges and universities, the teaching quality evaluation index system based on multi-evaluation subjects has been built. In the evaluation of teaching quality, however, all factors which affect the teaching quality should be considered. Therefore, the indexes that affect the teaching quality are manifold, and the content should be evaluated will be also very much. So that teaching quality evaluation is a problem of multi-variable and complex nonlinear [3]. Due to the degree of each factor affecting the teaching quality is different, it is difficult to evaluate the teaching quality using a precise mathematical model, and thus the traditional classification method cannot deal with the problem accurately.
Since artificial neural network (ANN) has the ability of nonlinear mapping, it provides a research method for nonlinear classification and pattern recognition. However, there are no rules to determine the parameters of neural network. If the parameter selection is improper, it will cause some defects in the practical application [4]. As a new intelligent search algorithm, the gravitational search algorithm (GSA) [5-6] has a strong global optimization capability. Just like other intelligent optimization algorithms, however, GSA also has the problem of poor local optimization ability. To overcome this shortcoming, the idea of chaotic sequence is introduced into GSA to improve the searching performance. Then an evaluation method of teaching quality based on BP neural network using chaotic gravitational search algorithm (CGSA) is proposed, and the effectiveness is verified by an example.

\section{GRAVITATIONAL SEARCH AlgORITHM}

Gravitational search algorithm [7] is a global random search algorithm which is proposed by Rashedi in 2009. It is a kind of intelligent optimization algorithm based on the law of universal gravitation, and has strong global optimization ability. In the GSA algorithm, the solution of the optimization problem is represented by the particle position. Due to the gravitational attraction of the other particles in the population, the particle will have an acceleration, which leads to the speed and position will be changed.

Suppose there are $\mathrm{N}$ particles in $\mathrm{D}$ dimension space, the position of the $i$-th particle is $x_{i}=\left(x_{i}^{1}, x_{i}^{2}, \cdots, x_{i}^{D}\right)$, where D is the dimension of the position vector $x_{i}(t)$. The mass $M_{i}(t)$ of the particle $i$ at the moment $t$ is:

$$
\begin{gathered}
m_{i}(t)=\frac{f_{i}(t)-\operatorname{worst}(t)}{\operatorname{best}(t)-\operatorname{worst}(t)} \\
M_{i}(t)=\frac{m_{i}(t)}{\sum_{j=1}^{N} m_{j}(t)}
\end{gathered}
$$

Where $\mathrm{f}_{\mathrm{i}}(\mathrm{t})$ is the fitness value of $i$-th particle at time $t$. In addition, to solve the problem of maximum function, the best and worst correspond to the maximum and minimum fitness 
values, respectively. On the contrary, they take the minimum and maximum fitness, respectively.

Using the Newton's second law, the formula of gravitational acceleration of $j$-th particle acting on the $i$-th particle is given as:

$$
a_{i j}(t)=\frac{F_{i}(t)}{M_{i}(t)}=G(t) \cdot \frac{M_{j}(t)}{R_{i j}(t)+\varepsilon}\left(x_{j}-x_{i}\right)
$$

Where $\mathrm{G}(\mathrm{t})$ denotes a gravitational constant, $\mathrm{R}_{\mathrm{ij}}(\mathrm{t})$ represents the Euclidean distance between $\mathrm{x}_{\mathrm{i}}$ and $\mathrm{x}_{\mathrm{j}}, \varepsilon$ is a very small number.

At this point, the velocity and position update formulas for the particle $i$ are expressed as follows:

$$
\begin{gathered}
v_{i}(t+1)=\operatorname{rand} \cdot v_{i}(t)+a_{i}(t) \\
x_{i}(t+1)=x_{i}(t)+v_{i}(t+1)
\end{gathered}
$$

Where rand is a uniformly distributed random number within the interval of $[0,1]$.

Since the initial population of GSA algorithm is randomly generated, it is easy to produce repeated and uneven distribution. Therefore, the chaotic sequence is introduced into GSA algorithm, and then the ergodicity of chaotic sequence is used to generate the initial population. In this paper, the chaotic sequence is chosen as [8]:

$$
x_{i, j}=x_{\min , j}+c h_{k, j} \times\left(x_{\max , j}-x_{\min , j}\right)
$$

Where $c h_{k, j}=\sin \left(\pi \cdot c h_{k-1, j}\right), c h_{k-1, j} \in(0,1)$ is a chaotic sequence.

\section{CGSABP ALGORITHM}

To improve the ability of BP network to deal with complex problems, the initial weights and threshold parameters of BP neural network are optimized by the CGSA algorithm. In the experiment, the mean square error (MSE) of network outputs is taken as the fitness function of CGSABP algorithm, the goal of network training is to minimize MSE. The implementation process of CGSABP algorithm is described as follows:

1) Determine the input and output variables of CGSABP algorithm, and then the training and test samples are established.

\section{2) Initialization of neural network and GSA parameters}

Determine the topology of BP neural network, and initialize CGSA parameters, such as the population size and the number of iterations.

3) The calculation of fitness function

In this work, the greater the fitness function value in the CGSABP method is, the smaller the error between the target output and actual output obtains.

\section{4) CGSA iterative optimization}

The particle acceleration, velocity and position updating are calculated according to the fitness function value, and judges whether or not the termination condition is satisfied.

\section{5) Constructing CGSABP model}

The initial parameters of BP network optimized by the CGSA algorithm are obtained, and then the training date is used to train the CGSABP model.

\section{6) Evaluation of teaching quality}

The test data is input to the trained GSABP model to carry on the teaching quality evaluation.

\section{EVALUATION OF TEACHING QuALity}

At present, the evaluation of classroom teaching quality mainly consists of 4 components, such as the assessment of supervisions of instruction, peer evaluation, teacher self-evaluation and student evaluation. The 4 evaluation subjects are in different positions, and the function of them is also different. Therefore, the evaluation methods and results of each subject are only part of the classroom teaching quality evaluation. They can only reflect one side of the problem, and cannot be simply equivalent to the whole picture of teaching quality.

The content of classroom teaching quality evaluation varies from simple to complex, but the general index system may involve several aspects. For example, teaching attitude, teaching content, teaching method, teaching effect and teaching and educating, etc. The evaluation method used in the current teaching quality evaluation system is the weighted average method, which the evaluation indexes are formulated by the relevant departments in advance, and the students score according to the evaluation indexes. Consequently, the weight of each index is multiplied, and then the teacher's classroom teaching quality evaluation can be achieved. Finally, the mean score of each teacher is obtained by means of the arithmetic average method, and the evaluation grade of teaching quality can be acquired.

According to the final results of classroom teaching quality evaluation, the four grades are chosen, such as excellent, good, qualified and failed. Each grade corresponds to the number 1, 2,3 or 4 which are act as the outputs of neural network. In this paper, the student evaluation system is taken as the research object which has 13 indexes, and they are used as the inputs of the CGSABP model, which are represented by X1-X13, the evaluation level $\mathrm{Y}$ is used as the output of the CGSABP model. According to the evaluation data of teaching quality at a certain university, 18 groups among them are selected as the training samples, and the remaining 12 groups are used as the test samples set, which are listed in Table 1. 
TABLE I. TEST SET

\begin{tabular}{|c|c|c|c|c|c|c|c|c|c|c|c|c|c|c|}
\hline NO & X1 & $\mathrm{X} 2$ & X3 & $\mathrm{X4}$ & X5 & X6 & X7 & X8 & X9 & X10 & X11 & $\mathrm{X} 12$ & X13 & $\bar{Y}$ \\
\hline 1 & 0.9 & 1.0 & 1.0 & 0.9 & 0.9 & 0.9 & 0.9 & 0.9 & 0.9 & 0.9 & 0.8 & 0.8 & 0.9 & 4 \\
\hline 2 & 0.8 & 0.9 & 1.0 & 0.9 & 0.9 & 0.8 & 0.8 & 0.9 & 0.9 & 0.9 & 0.8 & 0.7 & 0.8 & 4 \\
\hline 3 & 0.7 & 0.5 & 0.8 & 0.7 & 0.8 & 0.7 & 0.8 & 0.8 & 0.8 & 0.8 & 0.7 & 0.7 & 0.6 & 3 \\
\hline 4 & 0.6 & 0.5 & 0.7 & 0.6 & 0.6 & 0.6 & 0.6 & 0.6 & 0.6 & 0.6 & 0.5 & 0.5 & 0.6 & 2 \\
\hline 5 & 0.5 & 0.4 & 0.5 & 0.5 & 0.5 & 0.4 & 0.5 & 0.5 & 0.5 & 0.4 & 0.4 & 0.5 & 0.5 & 1 \\
\hline 6 & 0.9 & 1.0 & 1.0 & 1.0 & 0.9 & 1.0 & 1.0 & 0.9 & 1.0 & 1.0 & 1.0 & 0.9 & 1.0 & 4 \\
\hline 7 & 0.9 & 0.8 & 0.8 & 0.9 & 0.8 & 0.9 & 0.9 & 0.8 & 0.8 & 0.8 & 0.8 & 0.8 & 0.8 & 4 \\
\hline 8 & 0.7 & 0.6 & 0.8 & 0.6 & 0.8 & 0.7 & 0.7 & 0.6 & 0.8 & 0.7 & 0.7 & 0.7 & 0.8 & 3 \\
\hline 9 & 0.5 & 0.4 & 0.6 & 0.5 & 0.6 & 0.7 & 0.5 & 0.5 & 0.6 & 0.6 & 0.5 & 0.6 & 0.7 & 1 \\
\hline 10 & 0.6 & 0.8 & 0.8 & 0.6 & 0.8 & 0.8 & 0.7 & 0.8 & 0.8 & 0.8 & 0.7 & 0.8 & 0.8 & 3 \\
\hline 11 & 0.9 & 0.8 & 0.9 & 0.9 & 0.8 & 0.9 & 0.9 & 0.8 & 0.8 & 0.8 & 0.9 & 0.8 & 0.8 & 4 \\
\hline 12 & 0.7 & 0.6 & 0.7 & 0.6 & 0.6 & 0.8 & 0.8 & 0.7 & 0.7 & 0.8 & 0.8 & 0.6 & 0.9 & 2 \\
\hline
\end{tabular}

In order to evaluate the classification performance of the proposed CGSABP model, the GSABP, CGSABP and BP algorithms are used for comparison. In making the experiment, the population size $\mathrm{N}=30$, the maximum iteration number is set to 300 , network structure 13-16-4, learning rate 0.05 , gravitational constant $\mathrm{G}_{0}=100$. The evaluation results are shown in Table 2. In addition, the decimal portions of the evaluation results are rounded to facilitate the mapping, then the corresponding distribution map is plotted, which is shown in Fig.1.

TABLE II. EVALUATION RESULTS

\begin{tabular}{ccccc}
\hline \multirow{2}{*}{ No } & Actual & \multicolumn{3}{c}{ Evaluation results } \\
\cline { 3 - 5 } & value & CGSABP & GSABP & BP \\
\hline 1 & 4 & 3.9646 & 3.8585 & 4.1018 \\
2 & 4 & 3.2164 & 3.0655 & 3.1018 \\
3 & 3 & 2.9231 & 2.8692 & 2.6045 \\
4 & 2 & 1.9179 & 1.7084 & 1.6168 \\
5 & 1 & 1.0265 & 1.2281 & 0.9022 \\
6 & 4 & 3.8440 & 3.5758 & 4.2018 \\
7 & 4 & 4.2660 & 3.0640 & 3.6505 \\
8 & 3 & 3.1113 & 3.3339 & 2.9179 \\
9 & 1 & 1.0785 & 0.8752 & 1.3726 \\
10 & 3 & 3.0290 & 2.0869 & 2.8011 \\
11 & 4 & 3.8941 & 4.1764 & 3.4687 \\
12 & 2 & 2.0029 & 2.0058 & 1.8281 \\
\hline
\end{tabular}

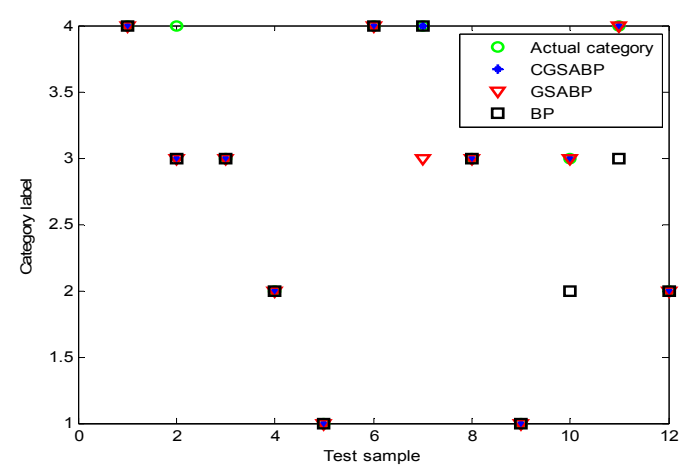

Fig. 1. Distribution of evaluation results

As can be seen from Table 2 and Fig.1, in the 3 teaching quality evaluation models in this paper, the CGSABP algorithm has a higher correct rate, reaching to $91.67 \%$, GSABP followed, its accuracy is $83.33 \%$, while the accuracy of BP algorithm is only $75 \%$. Obviously, although the CGSABP, GSABP and BP models can evaluate the quality of classroom teaching to some extent, but the CGSABP algorithm has better classification performance.

\section{CONCLUSIONS}

In this paper, an evaluation model of teaching quality based on GSABP algorithm is established, and the chaotic sequence is used to initialize the population, which improves the searching performance of GSA algorithm, and enhances the application ability of BP neural network. The evaluation examples show that the proposed method has better prediction effect than those of BP neural network and GSABP algorithm, and the generalization ability of the CGSABP model is stronger, it can provide a feasible method for the accurate evaluation of teaching quality.

\section{REFERENCES}

[1] Li Jianlin, Yao Yiyu, Nie Ming, et al, "ON TEACHING QUALITY EVALUATION SYSTEM OF THE UNIVERSITY TEACHERS BASED ON ROUGH SET THEORY," Computer Applications and Software, vol. 32, NO 3, pp. 59-63, 2014 (In Chinese).

[2] Wang Wei, Xu Hongqing, "The Strategies for Constructing a Teaching Evaluation System Against the Background of the Transformation in Local Colleges," Higher Education Forum, NO 11, pp. 76-78,91, 2014 (In Chinese).

[3] Wei Zhengyuan, Yan Kesheng, Su Yingying, "Model and Simulation of Maximum Entropy Neural Network for Teaching Quality Evaluation,", Computer Simulation, vol. 30, NO 5, pp. 284-287, 2013 (In Chinese).

[4] Cheng Jiatang, Duan Zhimei, XiongYan, "QAPSO-BP algorithm and its application in vibration fault diagnosis for a hydroelectric generating unit," Journal of Vibration and Shock, vol. 34, NO 23, pp. 177-181,201, 2015 (In Chinese).

[5] Liu Yongqian, Xu Qiang, David Infield, et al, "Fault identification of wind turbine drivetrain using BP neural network based on gravitational search algorithm," Journal of Vibration and Shock, vol. 34, NO 2, pp. 134-137, 2015 (In Chinese).

[6] Wang Yu, Huang Sheng, Liao Quanmi, et al, "A Method for Ship Compartment Layout Design Based on Gravitational Search Algorithm," Journal of Shanghai Jiao Tong University, vol. 50, NO 1, pp. 131-139, 2016 (In Chinese).

[7] Yuan Chao, Min Dai, Kai Chen, et al, "A novel gravitational search algorithm for multilevel image segmentation and its application on semiconductor packages vision inspection," Optik, NO 127, pp. 5770-5782, 2016.

[8] Li Huang, Shuai Ding, Shouhao Yu, et al, "Chaos-enhanced Cuckoo search optimization algorithms for global optimization," Applied Mathematical Modelling. NO 40, pp. 3860-3875, 2016. 